\title{
Development of a new crossbreed based evaluation for carcass quality of Piétrain boars in the Walloon Region of Belgium
}

M Dufrasne ${ }^{1}, \mathrm{H} \mathrm{Hammami}^{1}$, V Jaspart ${ }^{2}$, J Wavreille ${ }^{3}$, N Gengler ${ }^{1,4}$

${ }^{1}$ University of Liege Gembloux Agro-Bio Tech, Gembloux, Belgium, ${ }^{2}$ Walloon Pig Breeders Association, Ciney, Belgium, ${ }^{3}$ Walloon Agricultural Research Centre, Gembloux, Belgium, ${ }^{4}$ National Fund for Scientific Research, Brussels, Belgium

Email: Marie.Dufrasne@ulg.ac.be

Introduction Until recently, Piétrain boars in the Walloon Region were evaluated with performances recorded on their purebred progeny. However, these boars are mostly used in crossbreeding systems. Therefore, since 2007, a new genetic evaluation system has been developed in the Walloon Region. Piétrain boars are now evaluated on performances recorded on their crossbred progeny with Landrace sows. The aim of this study was to contribute to the new genetic evaluation system of these boars by the development of a genetic evaluation model for carcass quality. The objective was to develop a tool that allows selection of boars that produce carcass with a high lean meat percentage.

Material and methods Data provided by the on-farm performance recording system, also recorded at a central test station, were utilized in this study. Performances were recorded on live animals by ultrasound with the Piglog 105 . This strategy provided data recorded on animals from the test station, measured the week before slaughtering, and on boars themselves and other related animals on their farms of origin. The data file contains 60,546 records from pigs between 150 and 300 days of age, originated from 56822 different animals. Animals used needed to have a breed composition of at least $40 \%$ Piétrain or Landrace. Recorded animals were entire males, castrated males or females. Traits analysed in this study were backfat thickness (BF) and meat percentage (\%meat). The model developed was a multitrait animal model. Fixed effects were sex, contemporary groups and heterosis, modelled as regression on heterozygosity. A clustering algorithm created contemporary groups containing at least three animals measured at the same location in an interval of maximum 75 days. Random effects were additive genetic, permanent environment and residual. Additive genetic and permanent environment effects were modelled by random regressions using linear splines with three knots at 175, 200 and 250 days. Variance components were estimated by restricted maximum likelihood (REML) on random samples of the dataset and then confirmed by a Gibbs sampling algorithm on the total dataset. Fit of the models was tested by computing residuals from a BLUP (Best Linear Unbiased Prediction) evaluation. The model that could explain the greatest proportion of the variation in each trait and thus with the smallest residuals was selected. The t-test of Student was used to test whether the means of residual distributions were significantly different from zero.

Results Estimated heritabilities for $\mathrm{BF}$ and \%meat were high and had a tendency to increase with age. Estimated heritability from 150 to 300 days increased from 0.56 to 0.75 for $\mathrm{BF}$ and from 0.55 to 0.69 for \%meat. Genetic correlation was high between BF and \%meat and varied from -0.90 to -0.93 between 150 and 300 days. Figure 1 shows the evolution of mean residuals of each trait with age between 150 and 300 days. According to this figure, mean residual is close to zero for both traits at any age. The means of residual distributions of the two traits are not significantly different from zero (P Value $=0.7977$ for $\mathrm{BF}$ and $\mathrm{P}$ Value $=0.1476$ for $\%$ meat $)$.
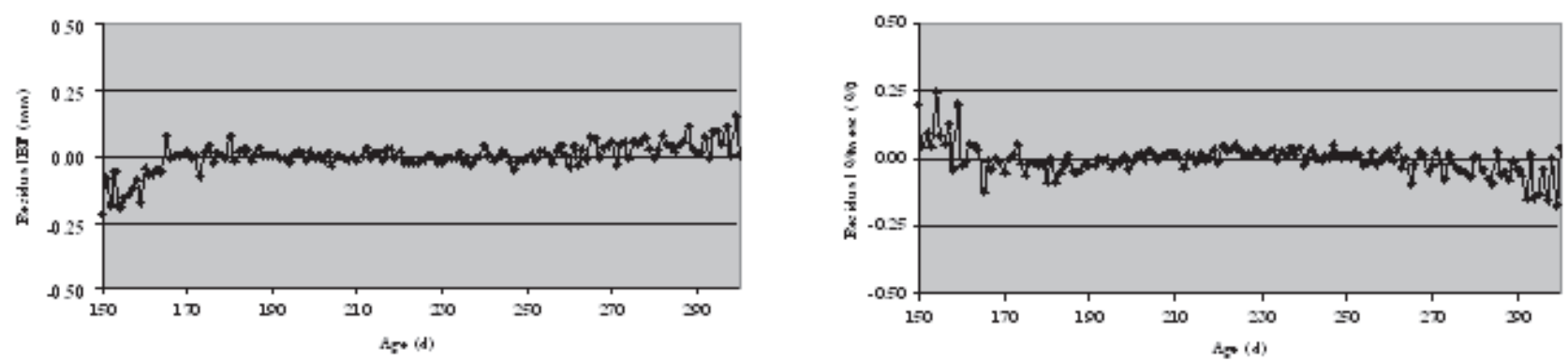

Figure 1 Evolution of mean residual with age between 150 and 300 days of age

Conclusions Given that BF and \%meat had a high heritability and a high genetic variance (results not shown), genetic improvement of carcass quality is possible by genetic selection with these traits. Moreover genetic correlation was high between $\mathrm{BF}$ and \%meat, so selection to increase \%meat could be based on only one of these two traits. Selection on BF would be preferred because it is a trait that is measured while \%meat is predicted from BF and loin muscle depth measurement by the Piglog 105. According to the study of residuals, it seems that the model developed fits well the data.

Acknowledgements The authors gratefully acknowledge the financial support of the Ministry of Agriculture of the Walloon Region of Belgium, the support of the Walloon Pig Breeders Association (AWEP) and the collaboration of the Walloon Agricultural Research Centre (CRA-W). Nicolas Gengler, a research associate of the National Fund for Scientific Research (Brussels, Belgium) acknowledges his support. Additional support was provided through grants 2.4507.02F (2) and F.4552.05 of the National Fund for Scientific Research. 